\title{
Efficient IP Table Lookup via Adaptive Stratified Trees with Selective Reconstructions
}

\author{
MARCO PELLEGRINI and GIORDANO FUSCO \\ Istituto di Informatica e Telematica \\ Consiglio Nazionale delle Ricerche \\ via Moruzzi 1, 56124 Pisa, Italy. \\ E-mail: \{marco.pellegrini, giordano.fusco\}@iit.cnr.it
}

IP address lookup is a critical operation for high bandwidth routers in packet switching networks such as Internet. The lookup is a non-trivial operation since it requires searching for the longest prefix, among those stored in a (large) given table, matching the IP address. Ever increasing routing tables size, traffic volume and links speed demand new and more efficient algorithms. Moreover, the imminent move to IPv6 128-bit addresses will soon require a rethinking of previous technical choices. This article describes a the new data structure for solving the IP table look up problem christened the Adaptive Stratified Tree (AST). The proposed solution is based on casting the problem in geometric terms and on repeated application of efficient local geometric optimization routines. Experiments with this approach have shown that in terms of storage, query time and update time the AST is at a par with state of the art algorithms based on data compression or string manipulations (and often it is better on some of the measured quantities).

Categories and Subject Descriptors: C.2.6 [Internetworking]: Routers

General Terms: Algorithms.

Additional Key Words and Phrases: IP Table lookup; data structures.

\section{INTRODUCTION}

Motivation for the Problem. Internet is surely one of the great scientific, technological and social successes of the last decade and an ever growing range of services rely on the efficiency of the underlying switching infrastructure. Thus improvements in the throughput of Internet routers are likely to have a large impact. The IP Address Lookup mechanism is a critical component of an Internet Packet Switch (see [McKeown 1999] for an overview). Briefly, a router within the network holds a lookup table with $n$ entries where each entry specifies a prefix (the maximum length $w$ of a prefix is 32 bits in the IPv4 protocol, 128 bits in the soon-to-bedeployed IPv6 protocol) and a next hop exit line. When a packet comes to the router, the destination address in the header of the packet is read, the longest prefix in the table matching the destination is sought, and the packet is sent to the corresponding next hop exit line. How to solve this problem so to be able to handle millions of packets per second has been the topic of a large number of research

Preliminary version in Proceedings of the 12th European Symposium on Algorithms (ESA). LNCS 3221, 2004.

Permission to make digital/hard copy of all or part of this material without fee for personal or classroom use provided that the copies are not made or distributed for profit or commercial advantage, the ACM copyright/server notice, the title of the publication, and its date appear, and notice is given that copying is by permission of the ACM, Inc. To copy otherwise, to republish, to post on servers, or to redistribute to lists requires prior specific permission and/or a fee.

(C) 20YY ACM 0004-5411/20YY/0100-0001 $\$ 5.00$ 
papers in the last 10-15 years. Steady increase in the size of the lookup tables and relentless demand of traffic performance put pressure on the research community to come up with faster schemes.

Our Contribution. In the IP protocol the address lookup operation is equivalent to the searching for the longest prefix match (lpm) of a fixed length string amongst a stored set of prefixes. It is well known that the longest prefix match problem can also be mapped into the problem of finding the shortest segment on a line containing a query point. This is called the geometric (or locus) view as opposed to the previous string view. The geometric approach has been proposed in [Warkhede et al. 2004] and [Lampson et al. 1999]; it has been extended in [Feldmann and Muthukrishnan 2000] and in several recent papers [Thorup 2003; Kaplan et al. 2003]. It has been used in [Buchsbaum et al. 2003] to prove formally the equivalence among several different problems. Important asymptotic worst case bounds have been found following the geometric point of view, but, currently, the algorithms for IP lookup with the best empirical performance, including the one in [Buchsbaum et al. 2003], are based on the string view and use either optimized tries, or data compression techniques, or both. The question that we rise is whether the geometric view is valuable for the IP lookup problem only as a theoretical tool or it is also a viable tool from a practical point of view. We notice that for the multi-dimensional packet filtering problem the multi-dimensional FIS tree [Feldmann and Muthukrishnan 2000], which follows the geometric view, has already established itself as a benchmark not only for the asymptotic bounds but also for empirical performance $^{1}$. Since our objective is practical we chose for the moment not to rely on the sophisticated techniques that have lead to the recent improvements in worst case bounds (e.g. those in [Feldmann and Muthukrishnan 2000; Thorup 2003; Kaplan et al. 2003] $)^{2}$. Instead, we ask ourselves whether simpler search trees built with the help of local optimization routines could lead to the stated goal. The experiments with the AST method lead us to give a strong positive hint on both accounts: (1) it is indeed possible to gain state of the art performance in IP lookup using the geometric view and (2) local adaptive optimization is a key ingredient for attaining this goal.

Since this is an experimental paper and the evaluation of the experimental data is critical we stress the experimental methodology beforehand in Section 2. In Section 3 we discuss previous work with the intent of highlighting the key ideas and key differences with the AST which is introduced in Section 4.1. In Section 4.2 we introduce some notation and preliminary storage reduction techniques, while in Section 5 the local optimization routines are described. Experiments and results for storage and query time are in Section 6 , while dynamic operations are discussed in Section 7. In Sections 8 and 9 we extend the review of related work to more recent results and compare them with the AST.

\footnotetext{
${ }^{1}$ See [Geraci et al. 2005] for a an alternative efficient method for multi-dimensional packet filtering based on geometric concepts.

${ }^{2}$ In Section 8 we comment of the relative practical performance of FIS tree and AST for IP lookup.

Journal of the ACM, Vol. V, No. N, Month 20YY.
} 


\section{METHODOLOGY}

Performance is established by means of experiments which are described in detail in Section 6. The experimental methodology we adopted follows quite closely the standards adopted in the papers describing the methods we compare to. Given the economic relevance of Internet technologies, such state of the art methods have been patented or are in the process of being patented, and, at the best of our knowledge, the corresponding codes (either as source or as executables) are not in the public domain. We refrained from re-implementing these methods, using as guidance the published algorithms, since we are aware that at this level of performance (few dozens of nanoseconds) overlooking seemingly simple implementation details could result in an grossly unfair comparisons. Given the above mentioned difficulties, we resolved for an admittedly weaker but still instructive comparison via the fact that those papers present, either an explicit formula for mapping their performance onto different machines ${ }^{3}$, or such formulae could be derived almost mechanically from the description in words. A second issue was deciding the data sets to be used. Papers from the late nineties did use table snapshots usually dating from the mid nineties, when a table of 40,000 entries was considered a large one. Although recovering such old data is probably feasible and using them certainly interesting, the evolution of Internet in recent years has been so rapid that the outcome of comparisons based on outdated test cases is certainly open to the criticism of not being relevant for the present (and the future) of Internet. Therefore the comparisons shown in Tables (IV) and (V), where we use data sets of comparable size and the mapping onto a common architecture given by the formulae, should be considered just in a broad qualitative sense. The main general conclusion we feel we can safely draw is that geometric view is as useful as insight drawn from string manipulation and data compression.

We remark that the extensive testing with 12 Lookup Tables of size ranging from 17,000 to 142,000 (reported in Tables (I), and (II)) confirms the reliability and robustness of the AST.

\section{PREVIOUS WORK}

The literature on the IP lookup problem and its higher dimensional extension ${ }^{4}$ is rather rich and a complete survey is beyond the scope of this article. Here we mention briefly the principles underlying some of the methods. At a high level we can distinguish three main approaches: (i) data structures and software searching techniques (ii) design of specialized hardware aiming at exploiting machine-level parallelism (iii) avoiding the lookup process altogether by exploiting additional header information. Here we comment only on approach (i).

\footnotetext{
${ }^{3}$ Naturally such formulae cannot capture all the nuisances of modern CPU architectures: they are based on the choice of a broad family (e.g. RISC vs CISC), leaving as parameters the clock frequency as well as size and access time of cache and RAM memory.

${ }^{4}$ Sometimes the IP lookup problem is treated as the 1-dimensional special case of the more general packet filtering problem. Similarly in geometric terms, the predecessor problem in 1-dimension can be seen as a special case of the (hyper)-rectangle stabbing problem in dimension $d \geq 2$. When the packet filtering is solved via dimensionality reduction, the IP-lookup can be thought of as the base case of the recursion. Sometimes the terminologies of the IP Lookup and the Packet Filtering problems get mixed up and this may generate some confusion.
} 
The classical data structure to solve the longest matching prefix problem is the binary trie [Knuth 1973]. Patricia Tries [Morrison 1968] take advantage of common sub-sequences to compress certain paths in a binary trie, thus saving both in search time and storage. The first IP Lookup algorithm in [Sklower 1991] is based on the Patricia Trie idea. Nilsson and Karlsson [Nilsson and Karlsson 1999] add the concept of level compression by increasing the out-degree of each node of the trie as long as at least a large fraction (called the filling factor) of the sub-trees of a node are non-empty.

Waldvogel et al. [Waldvogel et al. 1997] organize the prefixes in groups of prefixes of same length and each group is stored in a hash table for fast membership testing. The search is essentially a binary search by prefix length over the hash tables (a quite similar technique is in [Willard 1983]). The number of hash tables can be reduced by padding some of the prefixes [Srinivasan and Varghese 1999]. Good hashing strategies are studied in [Broder and Mitzenmacher 2001a].

In [Srinivasan and Varghese 1999] a method called variable stride trie is described. Here a trie is built but the number of bits (stride) used for the branching at a node is chosen separately for each trie node. The choices that optimize the memory occupation are found via dynamic programming. In [Buchsbaum et al. 2003] the trie is augmented by compressing all leaves and internal nodes via shortest common super-string compression [Blum et al. 1994]. It should be noticed that while in [Srinivasan and Varghese 1999] the model states a 1 Mbyte cap on storage and tries to attain the best worst case time within the cap (which is also our model), the emphasis in [Buchsbaum et al. 2003] is different: the target is a good average time for random traffic with no limit on storage, thus up to 3 Mbytes are used to store a table of $118 \mathrm{~K}$ entries.

Dagemark et al. [Degermark et al. 1997] use data compression techniques to store compactly parts of the prefix-tree representing the set of prefixes. At present this technique achieves in practice the lowest use of storage. Crescenzi et al. [Crescenzi et al. 1999] instead start from a full table representation of the lookup function then apply a data compression technique that reduces the storage to acceptable levels in practice while requiring only 3 memory accesses to answer a query.

The Multiway Range Tree [Warkhede et al. 2004] and the Multiway seach tree [Lampson et al. 1999] are the two early geometric based methods. The first one is akin to a classical segment tree with a root-to-leaf visit. The second one is akin to a fixed stride k-ways trie with a very large branching factor at the root. The branching degree is uniform and is decided beforehand; no optimization, local or global is done.

Ergun et al. [Sharp et al. 2001] and [Ergun et al. 2001] use the fast reconfiguration capabilities of skip lists [Pugh 1990] to adapt on-line the search data structure to the modifications of traffic patterns.

A number of recent papers have addressed the IP lookup problem with the aim of improving the worst case asymptotic performance. Dynamic 1-D Fat Inverted Segment Trees [Feldmann and Muthukrishnan 2000] is an elaboration of traditional segment trees and, for a small constant $l$, achieves query time $O(\log w+l)$ uses storage $O\left(n+l n^{1+1 / l}\right)$ and has amortized update cost $O\left(l n^{1 / l} \log n\right)$ over a sequence of $O\left(n^{1 / l}\right)$ updates. Thorup [Thorup 2003] improves the Dynamic FIS Tree: the

Journal of the ACM, Vol. V, No. N, Month 20 YY. 
storage becomes linear in $n$ and any sequence of dynamic operations is supported efficiently (a detailed discussion of the FIS tree approach is postponed to Section 8). Kaplan, Molad and Tarjan [Kaplan et al. 2003] give new algorithms for maintaining sets of intervals on a line that can be used for solving the IP lookup problem efficiently in the pointer model.

\section{THE AST}

\subsection{The AST in a nutshell.}

The AST construction algorithm is described in Section (5). Here we summarize the main new ideas. The best way to explain the gist of the algorithm is to visualize it geometrically. We first map the lookup problem into a predecessor search problem (see Section (4.2) for details). The equivalent input for the predecessor problem is a set of labelled points on the real line. We want to split this line into a grid of equal size buckets, and then proceed recursively and separately on the points contained in each grid bucket. A uniform grid is completely specified by giving an anchor point $a$ and the step $s$ of the grid. During the query, finding the bucket containing the query point $p$ is done easily in time $O(1)$ by evaluating the expression $\lfloor(p-a) / s\rfloor$ which gives the offset from the special bucket containing the anchor. We will take care of choosing $s$ as a power of 2 , so to reduce integer division to a right shift. If we choose the step $s$ too short we might end up with too many empty buckets, which implies a waste of storage. We choose thus $s$ as follows: choose the smallest $s$ for which the ratio of empty to occupied buckets is no more than a user-defined constant threshold. On the other hand shifting the grid (i.e. moving its anchor) can have dramatic effects on the number of empty buckets, occupied buckets and the maximum number of keys in a bucket. So the search for the optimal step size includes an inner optimization loop on the choice of the anchor to minimize the maximum bucket occupancy. The construction of the (locally) optimal step and anchor can be done efficiently in time close to linear, up to polylog terms (see Section 5). The algorithm works in two main phases, in the first phase we build a tree that aims at using small space at the expense of exhibiting a few long paths. In the second phase the long paths are shortened by compressing them, increasing the storage used.

During the construction a basic sub-task is finding a grid optimizing a local objective function. This approach has to be contrasted with several techniques in literature where a global optimality criterion is sought usually via much more expensive dynamic programming techniques, such as in [Cheung and McCanne 1999], [Gupta et al. 2000], [Srinivasan and Varghese 1999], and [Buchsbaum et al. 2003]. Ioannidis, Grama and Atallah [Ioannidis et al. 2005] recently proposed a global reconstruction method for tries that relies on a reduction to the knapsack problem and therefore suggests the use of knapsack approximation methods. In [Ioannidis et al. 2005] the target is to reduce the average query time based on traffic statistics, not the worst case query time.

A simple theoretical analysis of the asymptotic worst case query time/storage for the AST gives asymptotic worst case bounds $O(n)$ for storage and $O(\log n)$ for query time at the end of the first phase. We are not able to quantify asymptotically the improvements introduced in the second phase since the computation is 

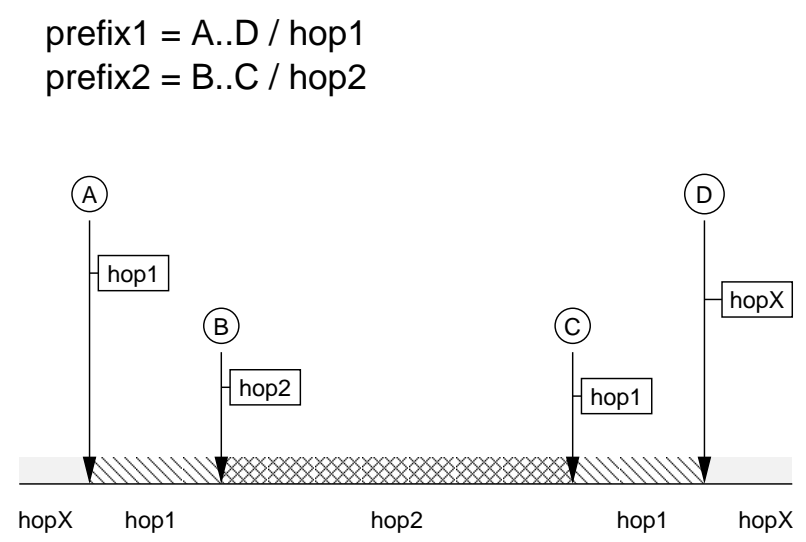

Fig. 1. Intervals and next-hops

essentially data adaptive. However the query time bound does not explain the observed performance and we leave it as an open problem to produce a more refined asymptotic analysis.

\subsection{The Problem}

The longest prefix match problem has an input that consists of a set $T$ of $0 / 1$ strings of length up to an integer $w$ and a function mapping each prefix to a label indicating the next-hop. For any destination address $q$ we return the label associated to the longest among the strings in $T$ that are prefixes of $q$. We can turn this problem into the shortest stabbing segment problem as follows. Each entry in a routing table has the form

$$
\text { netbase/netmask } \rightarrow \text { nexthop }
$$

where netbase is an 32-bits IP address, netmask denote the number of bits used for prefix and next-hop is the destination rout of the packets whose destination address matches the prefix specified in this entry. From each table entry we build a pair of values denoting the begin and the end of interval. The rule is:

$$
\begin{aligned}
\text { begin } & =\text { netbase } \wedge \text { mask } \\
\text { end } & =(\text { netbase } \vee(\neg \text { mask }))+1
\end{aligned}
$$

where $\wedge \vee \neg$ are the bitwise operators AND OR and NOT. Moreover we associate to begin and end values a label

$$
\begin{aligned}
\text { label[begin }] & =\text { nexthop } \\
\text { label }[\text { end }] & =\text { nexthop of previous matching prefix }
\end{aligned}
$$

Thus we can turn the set $T$ of labelled prefixes into a set $S$ of labelled points (see figure (1)). For any query point $q$, its predecessor point in $S$ is labelled correctly with the next-hop of the longest matching prefix for $q$. For a prefix $p \in T$, denote with $M(p)$ its corresponding segment (seen as a contiguous subset of the set $\left.\left[0, . ., 2^{w}-1\right]\right)$.

Semi-closed intervals. In real tables it happens quite often that the segments 
are adjacent, meaning that for two prefixes $p^{\prime}$ and $p^{\prime \prime}, \max M\left(p^{\prime}\right)+1=\min M\left(p^{\prime \prime}\right)$. In this case we can reduce considerably the number of required distinct points by considering our intervals closed on the left by open on the right. That is the pair $[a, b[$ with $b \geq a$ represents the interval $[a, b-1]$. In such a way the closed intervals $[a, b],[b+1, c]$ are represented as $[a, b+1[,[b+1, c+1[$ thus only 3 distinct points need to be recorded ${ }^{5}$ instead of 4 . The left end-point of the second interval is called a duplicate point.

Phantom points. If two consecutive points in $S$ have the same label, then the rightmost such point can be safely deleted from $S$ for the purpose of solving for static predecessor queries. As seen in Table (I) both duplicates and phantom represent a sizable portion of the points in actual tables.

\section{CONSTRUCTION OF THE AST}

\subsection{Main ideas}

A Generic Recursive Bucketing Tree. Here we describe the AST data structure by iteratively refining a general framework with specific choices. For simplicity we describe in detail the data structure that solves the predecessor problem in a set of points on the line. Afterwards we will comment on the small modifications needed to solve the dynamic IP-lookup problem.

We start by describing a Generic Recursive Bucketing Tree $T(U, S)$. Consider initially the set $U=\left[0, . ., 2^{w}-1\right]$ of all possible points and the input set $S \subset U$ of points. We build recursively a tree by levels. Each node $x$ of the tree has an associated connected subset of the universe $U_{x} \subseteq U$ (i.e an interval), and a point data set $S_{x} \subset S$, which is $S_{x}=S \cap U_{x}$. Initially at the root of the tree $r$ we have $U_{r}=U$ and $S_{r}=S$. Let $x$ be a node of the tree and let $k(x)$ be the number of children on $x$. By $y[1], \ldots, y[k(x)]$ we denote the children of $x$. We partition $U_{x}$ into a number $k(x)$ of disjoint intervals, $U_{1}, \ldots, U_{k(x)}$, each associated to a child of $x$, then as a consequence we associate to each child of $x$ the set of points $S_{y[i]}=U_{i} \cap S_{x}$. The recursive construction stops for a node $y$ when $\left|S_{y}\right| \leq c$ for a constant $c$. At each leaf $y$, we associate to $y$ the set $S_{y}$ and moreover the point in $S \backslash S_{y}$ that is the unique predecessor of any point in $U_{y}$.

How to query a Generic Recursive Bucketing Tree. When a query point $q$ is given, the Generic Recursive Bucketing Tree $T(U, S)$ is used to solve the predecessor problem as follows. Trace a path from root to leaf as follows: when node $x$ has been reached, visit the child $y$ of $x$ such that $q \in U_{y}$. When we reach a leaf $l$ at the end of this path, the answer is computed by direct comparison of $q$ with the $\left|S_{l}\right|+1$ points stored at leaf $l$.

From a Generic Recursive Bucketing Tree to an Adaptive Stratified Tree. The only step that need to be specified is: how do we compute the partition of $U_{x}$ into a number $k(x)$ of disjoint intervals? We use as split points the points of the

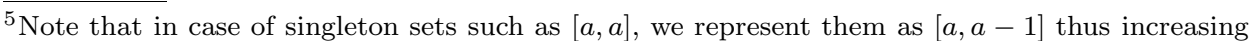
the number of points. In actual tables this increase happens rarely. If we use a machine with $w$-bit registers to store prefixes up to $w$ bits we might have to represent the number $2^{w}$ which require $w+1$ bits, this however is not a problem since for the purpose of a query we do not need to store the points beyond the query range, and for the purpose of updates we just treat the last number as a special case. 
infinite uniform grid $G(a, s)=\{a+k s: k \in N\}$ of anchor $a$ and step $s$ that fall in $U_{x}$ (that is, $U_{x} \cap G(a, s)$ ). Note that by using these split points all the induced intervals (buckets) are of equal length (that is $s$ ) except maybe the first and the last interval. Note also that finding the index of the bucket containing a query point $q$ amounts to just computing $\left\lfloor\frac{q-a}{s}\right\rfloor$, that is an $O(1)$ operation independent of the number of children of a node. Thus we just need to specify rules for choosing the anchor $a$ and the step $s$ for a node $x$. To do so we introduce two objective functions that we would like to minimize simultaneously. Since a multi-objective optimization is problematic, we will instead minimize one function subject to an upper bound on the other.

Let $E(a, s)$ be the number of buckets of the above construction containing no point in $S_{x}$, and $F(a, s)$ the number of buckets containing at least one point of $S_{x}$. The ratio is $R(a, s)=\frac{E(a, s)}{F(a, s)}$. Define also the minimum of such ratio over all possible choices of the anchor $a$ : $R_{\min }(s)=\min _{a} R(a, s)$. The ratio $R_{\min }(s)$ is the first objective function we are interested in. Such function has been defined with the purpose of controlling the number of empty buckets since a proliferation for empty buckets is bad for memory consumption. Full buckets instead contain input points therefore their number can be charged to the size of the input.

Let $G^{\prime}(a, s)$ be the set of intervals partitioning $U_{x}$ induced by $G(a, s) \cap U_{x}$. Consider $g(a, s)$, the maximum number of points of $S$ in any such interval, formally:

$$
g(a, s)=\max _{I \in G^{\prime}(a, s)}|S \cap I|
$$

Consider then the choice of anchor $a$ that minimizes this maximum number, thus leading to the following function: $g_{\min }(s)=\min _{a} g(a, s)$. The function $g_{\min }(s)$ is the second objective function we are interested in. Such function has been introduced with the purpose of controlling the maximum depth of the search tree.

Phase I: Saving Space. In order to control memory consumption we adopt the following action recursively and separately at each node: find smallest value $s^{\prime}$ such that $R_{\min }\left(s^{\prime}\right) \leq C$, for a predefined constant threshold $C$ then take an anchor $a$ that realizes the value of $g_{\min }\left(s^{\prime}\right)$, i.e. gives us the min-max occupancy (this shifting does not change the number of nodes at the level).

Phase II: Selective Reconstruction. The tree built in Phase I uses linear space (see proof below) but can have some root-to-leaf path of high length. The purpose of the second phase is to reduce the length of the long paths, without paying too much in storage. In order to do so we visit the longest paths and we decrease the value $s^{\prime}$ used in the split so to increase the number of children and decrease the min-max bucket occupancy. This operation trades off storage against tree depth.

\subsection{Further details of the construction}

How to compute efficiently $R_{\min }(s)$ and $g_{\min }(s)$. In the previous paragraphs we have introduced two functionals that we want to minimize and we have explained how they are used in the AST construction. Here we will show that such functions can be computed efficiently. Pre-processing time for data structures devoted to IP-lookup is not one of the most important parameters, however since occasionally one has to (partially) rebuild the data structures to maintain its properties, it is important that such operations are performed as efficiently as possible.

Journal of the ACM, Vol. V, No. N, Month 20YY. 
First of all we notice that by shifting the anchor by $s$ to the right, the grid remains unchanged: $G(a, s)=G(a+s, s)$. Consider the continuous movement of a grid: $f_{G}(\alpha)=G(a+\alpha s, s)$ for $\alpha \in[0,1]$. Call an event a value $\alpha_{i}$ for which $S \cap f_{G}\left(\alpha_{i}\right) \neq \emptyset$.

LEMMA 5.1. The number of events is at most n.

Proof. Take a single fixed interval $I$ of $G(a, s)$, we have an event when the moving left extreme meets a point in $I$, this can happen only once for each point in $I$. Therefore overall there are at most $n$ events.

Since we study bucket occupancy, extending the shift for $\alpha>1$ is useless since every distribution of points in the bucket has already been considered, given the periodic nature of the grid. Consider point $p \in S$ and the bucket $I_{p}$ containing $p$. Point $p$ produces an event when $\alpha_{p}=\left(p-\operatorname{left}\left(I_{p}\right)\right) / s$ that is when the shift is equal to the distance from the left extreme of the interval containing $p$. Thus we can generate the order of events by constructing a min-priority queue $Q\left(S, \alpha_{p}\right)$ on the set $S$ using as priority the value of $\alpha_{p}$. We can extract iteratively the minimum for the queue and update the counters $c(I)$ for the shifting interval $I$. Note that for our counters an event consists in decreasing the counter for a bucket and increasing it for the neighbor bucket. Moreover we keep the current maximum of the counters. To do so we keep a second max-priority queue $Q(I, c(I))$. When a counter increases, we apply the operation increase-key; when it decreases, we apply the operation decrease-key. Finally we record changes in the root of the priority queue, recording the minimum value found during the life-time of the algorithm. This value is the

$$
g_{\min }(s)=\min _{\alpha \in[0,1]} \max _{I \in G(\alpha s, s)}|S \cap I|
$$

that is we find the shift that for a given step $s$ minimizes the maximal occupancy. Using standard priority queue implementations, the whole algorithm for computing $g_{\min }(s)$ takes time $O(n \log n)$. Similarly we can compute $R_{\min }(s)$ within the same time bound. Next we show monotonicity properties of the functions $R_{\min }(s)$ and $g_{\min }(s)$ that will allow us to use binary search schemes in finding the value $s^{\prime}$.

Lemma 5.2. For any two step values $s$ and $t$, if $t=2 s$ then we have $g_{\min }(t) \geq$ $g_{\min }(s)$.

Proof. Consider the grid $G_{\min }(t)$ that attains min-max occupancy $K=g_{\min }(t)$. So every bucket in $G_{\min }(t)$ has at most $K$ elements. Now we consider the grid $G(s)$ that splits exactly in two every bucket in $G_{\min }(t)$. In this grid $G(s)$ the maximum occupancy is at most $K$, so the value $g(s)$ that minimizes the maximum occupancy for a translate of $G(s)$ cannot attain a larger value than $K$, i.e. $g_{\text {min }}(s) \leq K=g_{\text {min }}(t)$.

Lemma 5.3. For any two step values $s$ and $t$, if $t=2 s$ then we have $R_{\min }(t) \leq$ $R_{\min }(s)$.

Proof. Take the grid $G_{\min }(s)$, the grid of step $s$ minimizing the ratio $R_{\min }(s)=$ $E_{s} / F_{s}$. Now make the grid $G(t)$ by pairing adjacent buckets. Call $N_{s}, F_{s}, E_{s}$ the number of buckets, full buckets and empty buckets in $G_{\min }(s)$. Call $N_{t}, F_{t}, E_{t}$ the number of buckets, full buckets and empty buckets in $G(t)$. We have the relations: 
$N_{s}=N, N_{t}=N / 2, F_{s} / 2 \leq F_{t} \leq F_{s}$, and $\left(E_{s}-F_{s}\right) / 2 \leq E_{t} \leq E_{s} / 2$. Now we express $R_{t}=E_{t} / F_{t}$ as a function of $N$ and $F_{t}$.

$$
R_{t}=E_{t} / F_{t}=\left(\frac{1}{2}\right) N / F_{t}-1
$$

This is an arc of hyperbola (in the variable $F_{t}$ ) having maximum value for abscissa $F_{t}=F_{s} / 2$. The value of the maximum is $E_{s} / F_{s}=R_{\min }(s)$. Thus we have shown that $R(t) \leq R_{\min }(s)$. Naturally also $R_{\min }(t) \leq R(t)$ so we have proved $R_{\text {min }}(t) \leq R_{\text {min }}(s)$.

Thus the minimum ratio is monotonic increasing as grids get finer and finer and we can use binary search to find the largest step value satisfying the criterion of Phase I.

Details of the selective reconstruction. In current technology an access to the RAM memory is about ten times slower than one to L2 cache. Thus it is fundamental to fit all the relevant data into the L2 cache. It is reasonable to suppose that the target machine has, say, $1 \mathrm{Mb}$ or $2 \mathrm{Mb}$ of L2 cache and that it is completely devoted to the IP Table Lookup. With this hypothesis the size of the routing table does not make difference up to the L2 cache size. When the routing table is built, we can perform a selective reconstruction of the longest paths to flatten the AST in order to reduce the worst query time maintaining the total memory consumption below the L2 cache size. The following steps are repeated while the size of the Routing Table is below the L2 cache size and there are other reconstructions to perform:

(i) Create a max-priority queue of internal nodes based on the maximum cost of their children (see below).

(ii) Visit the AST and consider only internal nodes having only leaves as children: determine the cost of these nodes and insert them in the max-priority queue.

(iii) Extract from the queue the nodes with the same maximum cost and flatten them in the following way: if the maximum number of points in a child full leaf is greater than 1 then split the step until the maximum number of points in a bucket becomes smaller than the current maximum; otherwise go the parent and split its step until the maximum number of points in a bucket becomes smaller than maximum number of points a full leaf can contain (in this way the level below is eliminated).

A few exceptions to the above rules: the root is not rebuilt, and, for simplicity, when a level is eliminated other reconstructions of same cost are performed only after recomputing the priority queue.

The cost of a node. Each node of the AST has associated a cost which represents the time of the slowest query among all the queries that visit that node. The cost of a full leaf is the time to reach the last point stored inside it (i.e. the time to visit all the points from the median to the farthest extreme). The cost of an internal node is the maximum of the costs of all its children. See Table (III) for the costs in our experimental setting.

The leaves can be subdivided into classes (Lev, Pts, Base) depending on: their level Lev in the AST, the number of points Pts from the median to the farthest

Journal of the ACM, Vol. V, No. N, Month 20YY. 
extreme and the number of bits Base used to stored the points in the leaf $(8,16$ or $32)^{6}$.

To guide the selective reconstruction a Clock-ticks Table is build once for each CPU architecture. For all the possible classes of leaves, the table stores the corresponding query time. The query time of a particular class is measured in the following way: a simple AST containing nodes of the chosen class is built, several queries reaching nodes of that class are performed, the minimum time is taken and it is normalized to L2 cache. The rationale is that a machine dedicated to the IP Table Lookup is capable to perform a query in the minimum time measured in the test machine because the processor is not disturbed by other running processes. The measurements have been done using Pentium specific low level primitives to attain higher precision; nothing forbids to use operating system primitives for better portability.

Modifications for solving the IP-lookup problem. In order to solve the static IP-lookup method we just have to stored labelled active points instead of points. For the dynamic IP-lookup problem we need to store all labelled points (active, phantom and duplicates). Notice however that in all computations involving point/bucket occupancy only the active points will be considered, since duplicated and phantom points are relevant only for the update procedure. The root of the tree is treated in a special way since we use $2^{B}$ buckets. The maximum number of active points in a leaf is set to a parameter $D$. Finally the bound on the ratio $R_{\min }(s)$ is a constant $C$. The three parameters $B, C, D$ are specified by the user of the code at build time.

Asymptotic bounds for storage and worst case query time. Since at each node the number of empty buckets is bounded by the number of full buckets multiplied by a constant factor, it sufficed to analyze the number of full buckets. Since each full leaf has at least one point and each point is in at most one set $S_{l}$ for some leaf $l$, the number of full leaves is $O(n)$. Since by positioning the anchor at the median point of the set $S_{x}$ for a node $x$ we obtain at least two full children from any internal node, we have that the brunching factor of the tree restricted to the full nodes is always at least two, therefore the number of internal full nodes is linear in the number of full leaves. Thus the overall memory consumption is $O(n)$. Since at each level a bucket receives at most half of the points of its father, the depth is at most $O(\log n)$.

\section{EXPERIMENTS WITH AST}

Fat-AST versus Slim-AST. We consider two variants of the basic AST data structure. The Fat-AST is built on all input points (active, duplicates and phantom) in the data set, thus it can answer correctly any query and supports updates directly, however all occupancy counts are done with respect to the active points only. The Slim-AST is built only on the active points in a data set, thus it can answer correctly any query but is unable to support updates directly. Updates on the Slim-AST can be done either (i) by a partial execution of the building algorithm starting from the input data and portions of the data structure, or (ii) by requiring a version of

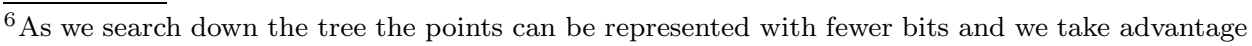
of this fact to decrease further the storage consumption by roughly $10 \%$.
} 


\begin{tabular}{|r|l||r||r|r||r|r|r|}
\hline & Routing Table & Date & Entries & $\begin{array}{c}\text { Total } \\
\text { Points }\end{array}$ & $\begin{array}{r}\text { Active } \\
\text { Points }\end{array}$ & $\begin{array}{c}\text { Phantom } \\
\text { Points }\end{array}$ & $\begin{array}{c}\text { Duplicate } \\
\text { Points }\end{array}$ \\
\hline 1 & Paix & $05 / 30 / 2001$ & 17,766 & 35,532 & 17,807 & 9,605 & 8,120 \\
2 & AADS & $05 / 30 / 2001$ & 32,505 & 65,010 & 32,642 & 17,071 & 15,297 \\
3 & Funet & $10 / 30 / 1997$ & 41,328 & 82,656 & 24,895 & 35,391 & 22,370 \\
4 & Pac Bell & $05 / 30 / 2001$ & 45,184 & 90,368 & 34,334 & 33,750 & 22,284 \\
5 & Mae West & $05 / 30 / 2001$ & 71,319 & 142,638 & 53,618 & 50,217 & 38,803 \\
\hline 6 & Telstra & $03 / 31 / 2001$ & 104,096 & 208,192 & 64,656 & 79,429 & 64,107 \\
7 & Oregon & $03 / 31 / 2001$ & 118,190 & 236,380 & 45,299 & 116,897 & 74,184 \\
8 & AT\&T US & $07 / 10 / 2003$ & 121,613 & 243,226 & 102,861 & 62,910 & 77,455 \\
9 & Telus & $07 / 10 / 2003$ & 126,282 & 252,564 & 85,564 & 86,738 & 80,262 \\
10 & AT\&T East Canada & $07 / 10 / 2003$ & 127,157 & 254,314 & 78,625 & 93,795 & 81,894 \\
11 & AT\&T West Canada & $07 / 10 / 2003$ & 127,248 & 254,496 & 78,708 & 93,824 & 81,964 \\
12 & Oregon & $07 / 10 / 2003$ & 142,475 & 284,950 & 108,780 & 84,165 & 92,005 \\
\hline
\end{tabular}

Table I. List of Input Lookup Tables. Input geometric statistics for active, duplicates and phantom points.

the Fat-AST for the input data on a different (usually slower and less expensive) memory bank and copying the portions of the tree that are changed back onto the Slim-Tree. For fairness we compare the Slim-AST against data structures that do not support fast updates like [Degermark et al. 1997] and [Crescenzi et al. 1999] (Table (IV)), and we compare the Fat-AST against data structures supporting also fast updates like the Variable Stride tries in [Srinivasan and Varghese 1999] (Table $(\mathrm{V}))$.

Worst case query time. The testing of the (empirical) worst case query time is done statically on the structure of the tree by finding out the longest (most expensive) path from root to leaf. The worst case query time gives valuable information that is relevant for, but independent from, any actual query data stream.

Table (I) gives a synthetic view of the IP Lookup Tables used for testing the AST data structure. For each table we report a progressive number, the name of the organization or router holding the table, the date when the Table was downloaded and the number of prefixes in the tables. Each prefix generates two points, next we classify the points into active, phantom and duplicate points, giving the count for each category.

Table (II) gives the relevant measure of storage, worst case memory access, and worst case time (in clock ticks) for the Slim-AST and the Fat-AST.

Normalization in L2. To test the AST data structure we adopt the methodology in [Degermark et al. 1997]. For completeness we describe the methodology. We visit each node of AST twice so to force data for the second invocation in L1 cache (This is known as the hot cache model). We measure number of clock-ticks by reading the appropriate internal CPU registers relative to the second invocation. As in [Degermark et al. 1997] we exclude from measurement the function invocation overhead and consider the search completed when we retrieve the index of the next-hop. We also record the number of L1 memory accesses of each query. This is important because, knowing the L1 latency, we decompose the measured time in CPU-operation cost and memory access cost. Afterwards we scale the memory access cost to the cost of accessing data in L2 cache, unless by locality we can argue that the data must be in L1 cache also on the target machine. We call this

Journal of the ACM, Vol. V, No. N, Month 20 YY. 


\begin{tabular}{|r|l||r||r|r|c|c|}
\hline & Routing Table & Entries & $\begin{array}{r}\text { Memory } \\
\text { Slim-AST }\end{array}$ & $\begin{array}{c}\text { Memory } \\
\text { Fat-AST }\end{array}$ & $\begin{array}{c}\text { Worst query } \\
\text { (clock-ticks) }\end{array}$ & $\begin{array}{c}\text { Worst Class } \\
\text { (Lev,Pts,Base) }\end{array}$ \\
\hline 1 & Paix & 17,766 & 374,314 & 440,436 & 35 & $2,2,8$ \\
2 & AADS & 32,505 & 460,516 & 589,762 & 35 & $2,2,8$ \\
3 & Funet & 41,328 & 453,034 & 679,568 & 30 & $2,1,8$ \\
4 & Pac Bell & 45,184 & 464,063 & 716,548 & 35 & $2,2,8$ \\
\hline 5 & Mae West & 71,319 & 610,587 & 980,182 & 35 & $2,2,8$ \\
6 & Telstra & 104,096 & 780,452 & $1,314,990$ & 35 & $2,2,16$ \\
7 & Oregon & 118,190 & 595,919 & $1,452,832$ & 35 & $2,3,8$ \\
8 & AT\&T US & 121,613 & 955,702 & $1,488,806$ & 30 & $2,1,8$ \\
9 & Telus (*) & 126,282 & 878,179 & $1,610,716$ & 40 & $2,4,8$ \\
10 & AT\&T East Canada & 127,157 & 878,155 & $1,542,682$ & 40 & $2,4,8$ \\
11 & AT\&T West Canada & 127,248 & 878,491 & $1,543,525$ & 40 & $2,4,8$ \\
12 & Oregon $(*)$ & 142,475 & 940,810 & $1,797,609$ & 35 & $2,2,16$ \\
\hline
\end{tabular}

Table II. Slim-AST data structure performance. Tests on a $700 \mathrm{MHz}$ Pentium III (T=1.4ns) with $1024 \mathrm{~KB} \mathrm{~L} 2$ Cache L2 delay is $\mathrm{D}=15 \mathrm{nsec}$, L1 latency $\mathrm{m}=4$ nsec. The parameters used are: $B=16 \quad C=10^{-7} \quad D=61$ for all tables except $(*)$ where $\quad C=1$.

\begin{tabular}{|c|c|c|c|r|c|}
\hline \multirow{2}{*}{ Position } & \multicolumn{5}{|c|}{ Level } \\
\cline { 2 - 6 } & 1 & 2 & 3 & \multicolumn{1}{|c|}{4} & 5 \\
\hline Empty & -3 & 20 & 42 & 66 & 116 \\
1 & 12 & 30 & 65 & 93 & 121 \\
2 & 12 & 35 & 67 & 94 & 125 \\
3 & 16 & 35 & 64 & 100 & 128 \\
4 & 22 & 40 & 69 & 101 & 129 \\
\hline
\end{tabular}

Table III. L2-normalized costs for points of 8 bits. Measures in clock ticks. The first negative entry is due to the inherent approximation in the measurement and normalization of the raw data; as an outlayer is not used in the construction of the interpolation formula.

measurement model the L2-normalized model. Since the root is accessed in each query, after unrolling the search loop, we store step, anchor and pointer to the first child at the root in registers so the access to the root is treated separately from accessing any other level of the AST. The results of the L2-normalized measures are shown in table (III) for 8-bit keys. Access times for 16 and 32 bit keys are almost identical.

Target architecture. Here we derive a formula we will use to scale our results to other architectures. The target architecture is made of one processor and two caches: one L1 cache and one L2 cache. $P \leftrightarrow C L 1 \leftrightarrow C L 2$. We suppose every memory access is an L1 miss, except when we access consecutive cells. We need to determine by fitting the experimental measurements the constants $a, b$ and $c$ in the formula for accessing a point at level $k$ and distance $s$ in the list stored at a leaf:

$$
a+k(M+m+b)+(M+m)+(s-1)(c+m),
$$

where $M$ is the time to access L2 cache, $m$ the time to access $T 1$ cache.

Since in Table (II) we have only results for classes from $(2,1)$ to $(2,4)$, in Table (III) we need accuracy of interpolation only in this range. Via easy calculations, the final interpolation formula for any $(2, s)$ class is: $3(M+m)+(s-1) m$. We denote with $D=M+m$ the L2 latency. 
Derivation of formulae in Tables (IV) and (V). From [Crescenzi et al. 1999, page 68]: "According to these measurements, on a Pentium II processor, each lookup requires $3 \cdot c l k+3 \cdot M_{D}$ nanoseconds, where $c l k$ denotes the clock cycle time and $M_{D}$ is the memory access delay". Thus in our notation this time formula becomes $3 T+3 D$, assuming the data structure fits completely in L2 memory.

From [Srinivasan and Varghese 1999, pages 27-29]. Several variants of the fixed and variable stride methods are compared on the 38816 prefixes Mae East table for different sizes of the L2 memory (512KB and $1 \mathrm{MB})$. Taking the data for the 1 MB L2 cache size in [Srinivasan and Varghese 1999, table X, page 29], the authors comment: "Now we consider the same database using a machine with $1 \mathrm{MB}$ of L2 cache (Table X). Here we see the minimum time is 148 nsec.; however this does not meet the worst-case deletion time requirements. In practice it might work quite well because the average insertion/deletion times are a few microseconds, compared to the worst case requirement of 2.5 msec." Since we are interesting in comparing at this stage only time versus storage, we do not consider the worst case update time as a drawback, and we concentrate on the method attaining the smallest query time while keeping the overall memory consumption below $1 \mathrm{MB}$. The entry that satisfies these requirements is attained for the algorithm "Leaf-pushed variable stride" with $k=3$ levels. In [Srinivasan and Varghese 1999, table VIII, page 27] this algorithm is characterized by the formula $(8 k+2) \cdot c l k+k \cdot M_{D}$, which, for $k=3$, in our notation gives the formula $26 T+3 D$.

From [Degermark et al. 1997, page 11]:" The difference in access time between the primary and the secondary caches is 20 nanoseconds (4 cycles). The lookup time on the Pentium Pro when two levels need to be examined is then at worst $69+8 \cdot 4=101$ cycles". Table [Degermark et al. 1997, table 2, page 10] reports 10 $\mathrm{ns}=2$ cycles L1 latency and 30ns $=6$ cycles for the Pentium Pro $200 \mathrm{MHz}$ machine used for the measurements. Since visiting each level of the data structure requires 4 memory accesses, two levels are accessed, and the latency of L2 access is 6 cycles we can derive $x=101-6 \cdot 8=53$ cycles for non-memory access operations and $8 D$ memory accesses, yielding a formula $53 T+8 D$. However from Table [Degermark et al. 1997, table 1, page 10] we see that a third level is often needed to handle all the queries in tables large enough. Thus a worst case estimate on the 32732 prefixes Mae East is given by adding 4 more memory access, yielding $53 T+12 D$. Anyhow, the qualitative conclusion we draw from the comparison do not change when either formula is used.

Discussion of AST performance. In Tables (IV) and (V) we compare the SlimAST and the Fat-AST methods with three other methods for which analogous formulae have been derived by the respective authors (explicitly or implicitly), mapped onto a common architecture. The method in [Degermark et al. 1997] is by far the most storage efficient, using roughly 4 bytes per entry; it is based on tree compression techniques, which make updates difficult. The query time in much higher than that of the AST. The Expansion/Compression method [Crescenzi et al. 1999] is very fast (requires only 3 memory access) using a fair amount of memory and requiring extensive reconstructions of the data structure at each update ${ }^{7}$ The Slim-

\footnotetext{
${ }^{7}$ However recently the average query time as well as the storage consumption have been somewhat improved (R. Grossi, personal communication).

Journal of the ACM, Vol. V, No. N, Month 20YY.
} 


\begin{tabular}{|l|l|r||r|r|r|}
\hline Ref & Method & $\begin{array}{r}\text { Input } \\
\text { size }\end{array}$ & Formula & $\begin{array}{r}\text { Time } \\
(\mathrm{ns})\end{array}$ & $\begin{array}{r}\text { Memory } \\
(\mathrm{KB})\end{array}$ \\
\hline [Degermark et al. 1997] & Lulea & 38141 & $53 \mathrm{~T}+12 \mathrm{D}$ & 254 & 160 \\
[Crescenzi et al. 1999] & Exp./Con. & 44024 & $3 \mathrm{~T}+3 \mathrm{D}$ & 49 & 1057 \\
This paper & Slim AST & 45184 & $\mathrm{~m}+3 \mathrm{D}$ & 49 & 464 \\
\hline
\end{tabular}

Table IV. Comparisons of static IP methods for a $700 \mathrm{MHz}$ Pentium III ( $\mathrm{T}=1.4 \mathrm{~ns}$ ) with $1024 \mathrm{~KB}$ L2 Cache L2 delay is $\mathrm{D}=15 \mathrm{nsec}$, L1 latency $\mathrm{m}=4 \mathrm{nsec}$.

\begin{tabular}{|l|l|r||r|r|r|}
\hline Ref & Method & $\begin{array}{r}\text { Input } \\
\text { size }\end{array}$ & Formula & $\begin{array}{r}\text { Time } \\
(\mathrm{ns})\end{array}$ & $\begin{array}{r}\text { Memory } \\
(\mathrm{KB})\end{array}$ \\
\hline [Srinivasan and Varghese 1999] & Variable stride & 38816 & $26 \mathrm{~T}+3 \mathrm{D}$ & 81 & 655 \\
This paper & Fat AST & 45184 & $\mathrm{~m}+3 \mathrm{D}$ & 49 & 716 \\
\hline
\end{tabular}

Table V. Comparisons of dynamic IP methods for a $700 \mathrm{MHz}$ Pentium III (T=1.4ns) with 1024 KB L2 Cache L2 delay is $\mathrm{D}=15 \mathrm{nsec}$, L1 latency $\mathrm{m}=4 \mathrm{nsec}$.

AST is as fast, but requires much less memory thus has better scaling properties.

The method of [Srinivasan and Varghese 1999] balances well query time, storage and update time. Its storage performance for tables of roughly 40,000 entries is not far form the one of the Fat-AST, however the Fat-AST is remarkably faster on a similar data set. Recent improvements of the scheme in [Srinivasan and Varghese 1999] are described and compared to the AST in section 8.

\section{DYNAMIC OPERATIONS}

Relative importance of performance measures. In this paper we designed the AST experiments so to give highest importance to the worst case query time, second in importance comes storage consumption, and finally comes update times. In this section we describe the dynamic operations on a Fat-AST. We describe some measurements on update time and we compare with those of a Variable Stride Tries in [Srinivasan and Varghese 1999]. Other dynamic ip lookup results are mentioned in Section 8 and compared to the AST.

Description of the dynamic operations. We give a brief sketch of the dynamic operations. Inserting a new prefix $p$ involves two phases: (1) inserting the endpoints of the corresponding segment $M(p) ;(2)$ updating the next-hop index for all the leaves that are covered by the segment $M(p)$. While phase (1) involves only two searches and local restructuring, phase (2) involves a DFS of the portion of the AST tree dominated by the inserted/deleted segment. Such a DFS is potentially an expensive operation, requiring in the worst case the visit of the whole tree. However we can trim the search by observing that it is not necessary to visit sub-trees with a span included into a segment that is included in $M(p)$ since these nodes do not change the next hop. There are $n$ prefixes and $O(n)$ leaves, and the span of each leaf is intersected without being enclosed by at most $2 D+1$ segments, therefore only $O(1)$ leaves need to be visited on average and eventually updated ${ }^{8}$.

$\overline{{ }^{8} \text { This rough }}$ argument can be made precise in an amortized sense by using the model of $(N, \Delta)$ - 


\begin{tabular}{|r|l||r|r|r||r|r|r||r|r|r|}
\hline \multicolumn{1}{|l|}{} & \multirow{2}{*}{ Routing Table } & \multicolumn{3}{c||}{$100 \%$} & \multicolumn{3}{c||}{$99 \%$} & \multicolumn{3}{c|}{$95 \%$} \\
\cline { 2 - 10 } & & Accesses & \multicolumn{1}{|c||}{ Time } & \multicolumn{2}{|c|}{ Accesses } & Time & \multicolumn{2}{|c|}{ Accesses } & Time \\
& & L2 & L1 & $\mu$-sec & L2 & L1 & $\mu$-sec & L2 & L1 & $\mu$-sec \\
\hline 1 & Paix & 107 & 734 & 4.54 & 97 & 668 & 4.13 & 43 & 288 & 1.80 \\
2 & AADS & 588 & 6 & 8.84 & 69 & 466 & 2.90 & 33 & 216 & 1.36 \\
3 & Funet & 4285 & 156 & 64.90 & 79 & 538 & 3.34 & 59 & 398 & 2.48 \\
4 & PacBell & 1296 & 154 & 20.06 & 71 & 480 & 2.98 & 35 & 222 & 1.41 \\
5 & MaeWest & 1941 & 84 & 29.45 & 103 & 706 & 4.37 & 51 & 346 & 2.15 \\
6 & Telstra & 1867 & 548 & 30.20 & 81 & 546 & 3.40 & 53 & 356 & 2.22 \\
7 & Oregon & 4381 & 1598 & 72.11 & 457 & 3184 & 19.59 & 87 & 588 & 3.66 \\
8 & AT\&T US & 1675 & 76 & 25.43 & 67 & 460 & 2.85 & 43 & 282 & 1.77 \\
9 & Telus & 1686 & 10 & 25.33 & 73 & 488 & 3.05 & 45 & 294 & 1.85 \\
10 & AT\&T East Canada & 1686 & 10 & 25.33 & 81 & 552 & 3.42 & 49 & 322 & 2.02 \\
11 & AT\&T West Canada & 1686 & 10 & 25.33 & 81 & 552 & 3.42 & 49 & 322 & 2.02 \\
12 & Oregon & 2309 & 76 & 34.94 & 465 & 3244 & 19.95 & 67 & 446 & 2.79 \\
\hline
\end{tabular}

Table VI. Fat-AST data structure performance of insertion. Number of memory access in L2 and L1 cache for the 100, 99 and 95 percentiles. Time estimate in the test machine. Tests on a 700 $\mathrm{MHz}$ Pentium III ( $\mathrm{T}=1.4 \mathrm{~ns})$ with $1024 \mathrm{~KB} \mathrm{~L} 2$ Cache L2 delay is $\mathrm{D}=15 \mathrm{nsec}$, L1 latency $\mathrm{m}=4$ nsec.

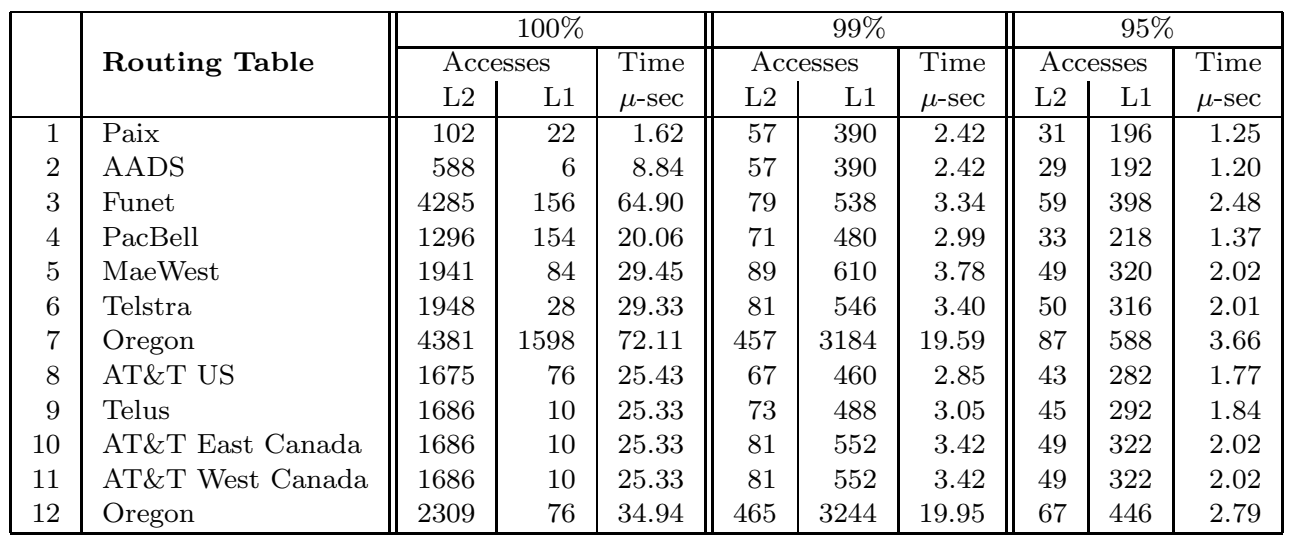

Table VII. Slim-AST data structure performance of deletions. Number of memory access in L2 and $\mathrm{L} 1$ cache for the 100, 99 and 95 percentiles. Tests on a $700 \mathrm{MHz}$ Pentium III (T=1.4ns) with $1024 \mathrm{~KB}$ L2 Cache L2 delay is $\mathrm{D}=15 \mathrm{nsec}$, L1 latency $\mathrm{m}=4$ nsec.

Deleting prefix $p$ from the AST involves phases similar to insertion, however we need to determine first the segment in the AST including $M(p)$ in order to perform the leaf re-labeling. This is done by performing at most $w$ searches, one for each prefix of $p$, and selecting the longest such prefix present in the AST. In practice fewer than $w$ searches are necessary in most cases. Although it is possible to check and enforce the other AST invariants at each update we have noticed that the query performance remains stable over long sequences of random updates. Therefore enforcing the AST invariant is best left to an off-line periodic restructuring process. Experimental data. We perform experiments on updating the AST using as

sequences of updates of Mulmuley [Mulmuley 1993].

Journal of the ACM, Vol. V, No. N, Month 20YY. 


\begin{tabular}{|c|c|c|c|c|c|c|c|c|c|c|}
\hline \multirow{3}{*}{$\begin{array}{l}\text { Prefix } \\
\text { Length }\end{array}$} & \multirow{3}{*}{$\begin{array}{c}\text { \# of } \\
\text { Prefixes }\end{array}$} & \multicolumn{3}{|c|}{$100 \%$} & \multicolumn{3}{|c|}{$99 \%$} & \multicolumn{3}{|c|}{$95 \%$} \\
\hline & & \multicolumn{2}{|c|}{ Accesses } & \multirow{2}{*}{$\begin{array}{l}\text { Time } \\
\mu \text {-sec }\end{array}$} & \multicolumn{2}{|c|}{ Accesses } & \multirow{2}{*}{$\begin{array}{l}\text { Time } \\
\mu \text {-sec }\end{array}$} & \multicolumn{2}{|c|}{ Accesses } & \multirow{2}{*}{$\begin{array}{l}\text { Time } \\
\mu \text {-sec }\end{array}$} \\
\hline & & L2 & L1 & & L2 & L1 & & L2 & L1 & \\
\hline 8 & 17 & 1686 & 14 & 25.35 & 100 & 0 & 1.50 & 100 & 0 & 1.50 \\
\hline 9 & 5 & 49 & 0 & 0.73 & 39 & 2 & 0.59 & 39 & 2 & 0.59 \\
\hline 10 & 8 & 1675 & 80 & 25.45 & 41 & 30 & 0.73 & 41 & 30 & 0.73 \\
\hline 11 & 13 & 1273 & 258 & 20.13 & 1223 & 60 & 18.59 & 1223 & 60 & 18.59 \\
\hline 12 & 54 & 841 & 122 & 13.10 & 744 & 140 & 11.72 & 479 & 142 & 7.75 \\
\hline 13 & 97 & 494 & 260 & 8.45 & 473 & 16 & 7.16 & 245 & 192 & 4.44 \\
\hline 14 & 259 & 251 & 382 & 5.29 & 204 & 286 & 4.20 & 124 & 174 & 2.56 \\
\hline 15 & 473 & 176 & 764 & 5.70 & 112 & 8 & 1.71 & 64 & 222 & 1.85 \\
\hline 16 & 7459 & 185 & 52 & 2.98 & 46 & 292 & 1.86 & 20 & 98 & 0.69 \\
\hline 17 & 1601 & 92 & 612 & 3.83 & 49 & 334 & 2.07 & 30 & 184 & 1.19 \\
\hline 18 & 2933 & 512 & 14 & 7.74 & 54 & 340 & 2.17 & 31 & 194 & 1.24 \\
\hline 19 & 8477 & 316 & 38 & 4.89 & 53 & 356 & 2.22 & 31 & 198 & 1.26 \\
\hline 20 & 8424 & 175 & 70 & 2.90 & 53 & 360 & 2.23 & 33 & 208 & 1.33 \\
\hline 21 & 5972 & 110 & 740 & 4.61 & 63 & 432 & 2.67 & 41 & 276 & 1.72 \\
\hline 22 & 8979 & 157 & 1080 & 6.67 & 65 & 436 & 2.72 & 49 & 326 & 2.04 \\
\hline 23 & 10088 & 157 & 1080 & 6.67 & 79 & 534 & 3.32 & 55 & 368 & 2.30 \\
\hline 24 & 67865 & 157 & 1080 & 6.67 & 87 & 588 & 3.66 & 57 & 386 & 2.40 \\
\hline 25 & 151 & 157 & 1080 & 6.67 & 157 & 1080 & 6.67 & 157 & 1080 & 6.67 \\
\hline 26 & 169 & 47 & 314 & 1.96 & 37 & 248 & 1.55 & 33 & 214 & 1.35 \\
\hline 27 & 696 & 157 & 1080 & 6.67 & 157 & 1080 & 6.67 & 157 & 1080 & 6.67 \\
\hline 28 & 691 & 48 & 306 & 1.94 & 47 & 314 & 1.96 & 33 & 214 & 1.35 \\
\hline 29 & 2490 & 157 & 1080 & 6.67 & 157 & 1080 & 6.67 & 37 & 248 & 1.55 \\
\hline 30 & 324 & 48 & 294 & 1.90 & 39 & 250 & 1.58 & 37 & 238 & 1.51 \\
\hline 31 & 0 & & & & & & & & & \\
\hline 32 & 3 & 39 & 250 & 1.58 & 29 & 180 & 1.16 & 29 & 180 & 1.16 \\
\hline
\end{tabular}

Table VIII. Fat-AST data structure performance of insertion by prefix length for Data set West AT\&T Canada. Number of memory access in L2 and L1 cache for the 100, 99 and 95 percentiles. Time estimate in the test machine. Tests on a $700 \mathrm{MHz}$ Pentium III ( $\mathrm{T}=1.4 \mathrm{~ns})$ with $1024 \mathrm{~KB}$ L2 Cache $\mathrm{L} 2$ delay is $\mathrm{D}=15 \mathrm{nsec}$, L1 latency $\mathrm{m}=4 \mathrm{nsec}$.

update data stream the full content of each table. For deletions this is natural since we can delete only prefixes present in the table. For insertions, we argue that entries in the table have been inserted in the past, thus it is reasonable to use them as representative also for the future updates ${ }^{9}$.

Tables (VI) and (VII) show upper estimates on the number of memory accesses and the percentile time in $\mu$-seconds for completing $95 \%, 99 \%$ and $100 \%$ of the updates (insertions and deletions) for the Fat-AST data structures built for the lookup tests. Note that experiments for deletions and insertion give very similar counts.

During the update we count the number of memory cells accessed distinguishing access in L2 and those in L1, assuming the data structure is stored completely in L2 cache. Since in the counting we adopt a conservative policy, i.e. we upper bound the number of cells accessed at each node visited during the update, the final count represents an upper bound on the actual number of memory access.

Tables (VIII) and (IX) show the insert and times (upper estimates) for a typical

\footnotetext{
${ }^{9}$ In public repositories of Internet data sets it seems to be difficult to find both table snapshots and traffic streams for the same tables, which would form an even better test case.
} 


\begin{tabular}{|c|c|c|c|c|c|c|c|c|c|c|}
\hline \multirow{3}{*}{$\begin{array}{l}\text { Prefix } \\
\text { Length }\end{array}$} & \multirow{3}{*}{$\begin{array}{c}\text { \# of } \\
\text { Prefixes }\end{array}$} & \multicolumn{3}{|c|}{$100 \%$} & \multicolumn{3}{|c|}{$99 \%$} & \multicolumn{3}{|c|}{$95 \%$} \\
\hline & & \multicolumn{2}{|c|}{ Accesses } & \multirow{2}{*}{$\begin{array}{l}\text { Time } \\
\mu \text {-sec }\end{array}$} & \multicolumn{2}{|c|}{ Accesses } & \multirow{2}{*}{$\begin{array}{l}\text { Time } \\
\mu \text {-sec }\end{array}$} & \multicolumn{2}{|c|}{ Accesses } & \multirow{2}{*}{$\begin{array}{l}\text { Time } \\
\mu \text {-sec }\end{array}$} \\
\hline & & L2 & L1 & & L2 & L1 & & $\mathrm{L} 2$ & L1 & \\
\hline 8 & 17 & 1686 & 10 & 25.33 & 96 & $\overline{0}$ & 1.44 & 96 & 0 & 1.44 \\
\hline 9 & 5 & 45 & 0 & 0.68 & 37 & 0 & 0.56 & 37 & 0 & 0.56 \\
\hline 10 & 8 & 1675 & 76 & 25.43 & 41 & 26 & 0.72 & 41 & 26 & 0.72 \\
\hline 11 & 13 & 1273 & 254 & 20.11 & 1223 & 56 & 18.57 & 1223 & 56 & 18.57 \\
\hline 12 & 54 & 841 & 118 & 13.09 & 742 & 138 & 11.68 & 477 & 140 & 7.71 \\
\hline 13 & 97 & 494 & 256 & 8.43 & 429 & 156 & 7.06 & 243 & 190 & 4.41 \\
\hline 14 & 259 & 251 & 378 & 5.28 & 204 & 282 & 4.19 & 122 & 32 & 1.96 \\
\hline 15 & 473 & 176 & 760 & 5.68 & 104 & 108 & 1.99 & 62 & 94 & 1.31 \\
\hline 16 & 7459 & 160 & 1080 & 6.72 & 43 & 272 & 1.73 & 20 & 100 & 0.70 \\
\hline 17 & 1601 & 90 & 610 & 3.79 & 47 & 310 & 1.95 & 30 & 172 & 1.14 \\
\hline 18 & 2933 & 512 & 10 & 7.72 & 54 & 336 & 2.15 & 31 & 208 & 1.30 \\
\hline 19 & 8477 & 316 & 34 & 4.88 & 53 & 360 & 2.23 & 31 & 198 & 1.26 \\
\hline 20 & 8424 & 175 & 66 & 2.89 & 53 & 360 & 2.23 & 33 & 220 & 1.38 \\
\hline 21 & 5972 & 110 & 736 & 4.59 & 63 & 428 & 2.66 & 41 & 272 & 1.70 \\
\hline 22 & 8979 & 157 & 1076 & 6.66 & 67 & 460 & 2.85 & 49 & 322 & 2.02 \\
\hline 23 & 10088 & 157 & 1076 & 6.66 & 79 & 530 & 3.31 & 55 & 368 & 2.30 \\
\hline 24 & 67865 & 157 & 1076 & 6.66 & 85 & 586 & 3.62 & 55 & 366 & 2.29 \\
\hline 25 & 151 & 157 & 1076 & 6.66 & 157 & 1076 & 6.66 & 157 & 1076 & 6.66 \\
\hline 26 & 169 & 47 & 310 & 1.95 & 37 & 244 & 1.53 & 33 & 210 & 1.33 \\
\hline 27 & 696 & 157 & 1076 & 6.66 & 157 & 1076 & 6.66 & 157 & 1076 & 6.66 \\
\hline 28 & 691 & 48 & 302 & 1.93 & 47 & 310 & 1.95 & 33 & 210 & 1.33 \\
\hline 29 & 2490 & 157 & 1076 & 6.66 & 157 & 1076 & 6.66 & 37 & 244 & 1.53 \\
\hline 30 & 324 & 48 & 290 & 1.88 & 37 & 248 & 1.55 & 35 & 236 & 1.47 \\
\hline 31 & 0 & & & & & & & & & \\
\hline 32 & 3 & 37 & 248 & 1.55 & 27 & 178 & 1.12 & 27 & 178 & 1.12 \\
\hline
\end{tabular}

Table IX. Fat-AST data structure performance of deletion by prefix length for Data set West AT\&T Canada. Number of memory access in L2 and L1 cache for the 100, 99 and 95 percentiles. Time estimate in the test machine. Tests on a $700 \mathrm{MHz}$ Pentium III ( $\mathrm{T}=1.4 \mathrm{~ns})$ with $1024 \mathrm{~KB}$ L2 Cache L2 delay is $\mathrm{D}=15 \mathrm{nsec}, \mathrm{L} 1$ latency $\mathrm{m}=4 \mathrm{nsec}$.

large table (West AT\&T Canada) with results separated by prefix length. For the same prefix length in both tables we obtain very similar counts. Note that the worst update time is attained by a few quite short prefixes (of length from 8 to 12).

Comparison of update time with [Srinivasan and Varghese 1999]. In [Srinivasan and Varghese 1999] an estimate of $2500 \mu$-sec for worst case updates is given counting the dominant cost of the memory accesses. The cost is incurred when a node in the trie with a large out degree $\left(2^{17}\right)$ needs to be rebuilt, and an access cost of 20 nsec per entry is assumed. Since 24-bit prefixes are very frequent (about $50 \%$ of the entries) the variable stride tries are forced so that updating for such length is faster, requiring roughly $3 \mu$-seconds. Although our machine is faster than that used in [Srinivasan and Varghese 1999] the L2 access time is quite similar, therefore the timings in [Srinivasan and Varghese 1999] can be compared (although only on a qualitative basis) with results in table (VI). We believe that the $2500 \mu$ sec worst case estimate for [Srinivasan and Varghese 1999] is overly pessimistic: in our experiments (deletion and insertion of every entry of every table), except for the Oregon data sets, up to $95 \%$ of the updates are completed in about $4 \mu$-seconds, thus

Journal of the ACM, Vol. V, No. N, Month 20 YY. 
very close to the average update time in [Srinivasan and Varghese 1999]. Globally the update performance of AST and Variable stride tries are comparable.

Finally we remark that updates in [Degermark et al. 1997] and [Crescenzi et al. 1999] are handled by rebuilding the data structure from scratch, thus requiring time of the order of several milliseconds for every update.

\section{MORE RELATED WORK AND COMPARISONS WITH AST}

Comparing the AST and the FIS tree. Let $S$ be the set of end-points of the input intervals, and $q$ a query point, we want to report the shortest segment in the input set containing $q$. If $q \notin S$ then the answer for $q$ is the same as the answer for the predecessor of $q$ in $S$, which is a point of $S$. If $q \in S$ we just have to recognize this fact, thus a dynamic dictionary is needed, and use an auxiliary data structure for a restricted form of the problem in which the query set coincides with $S$. Usually data structure for the predecessor problem is able to act as a dictionary. Thus we can solve the stabbing problem by using two data structures:

(I) A Data structure for Dynamic predecessor problem (in [Thorup 2003] also called Dynamic searching problem).

(II) A data structure for Dynamic list stabbing, with the restriction that the set of possible queries coincide with the set of end-points of the input segment.

The FIS Tree (Fat Inverted Segment Tree) is an elaboration of the segment tree and is used to implement the data structure specified in (II). Since the FIS-Tree has a tree structure and each end-point of the input segments is stored at a leaf of the FIS tree, we can couple data structures (I) and (II) using an inverse mapping that maps the points returned by the data structure (I) to the corresponding leaves of the data structure (II). This mapping operation is essential since the FIS-tree can be visited efficiently at query time only when the visit follows a leaf-to-root path (for this reason the adjective "Inverted" is part of the acronym).

Data structure (I) can be implemented using a variety of solutions in literature. In [Feldmann and Muthukrishnan 2000] it is suggested to use a dynamic van Emde Boas tree augmented with Dynamic Hashing so to keep the query time at $O(\log \log U)$ and reduce the storage to $O(n)$. A more practical solution also mentioned in [Feldmann and Muthukrishnan 2000] is that of using $B$-trees.

The coupling of the data structures (I) and (II) (e.g. van Emde Boas Tree + FIStree) is traversed as follows. First the da structure (I) is traversed in a root-to-leaf fashion so to identify the proper starting leaf in the data structure (II), afterwards the FIS tree is traversed from leaf-to-root, and candidate answers are collected at the nodes on the path, and among these the final answer is selected. If one were interested in a static solution, all this construction is useless and a data structure (I) with labelled points would be sufficient. So the coupling of the data structures (I) and (II) is needed in order to have dynamic update procedures with provable asymptotic performance.

The AST is not a FIS-tree. In particular the AST is a tree that is traversed in a root-to-leaf fashion and finds the correct output as a label of the reached leaf. Thus the AST sacrifices provable update performance bounds (and relies on empirically assessed measurements for this measure of quality) but gains in the fact that a 
single shallow tree has to be traversed. Even if a depth parameter $t=2$ is chosen for the FIS-tree, the twin data structure (I) when implemented as a van Emde Boas tree would visit $\log \log U$ nodes, thus for $U=2^{32}$, an additional 5 nodes are visited, giving a total of 7 visited nodes to answer any query. In our experiments with AST we would solve ip-lookup queries by visiting only two nodes of an AST tree and a total of three memory accesses. Since visiting a path in a tree would require at least a memory access per node, even without further experimental evidence, it is safe to conclude that any standard implementation of a FIS-tree based data structure for IP-lookup would result in a slower query time than the AST (or other specialized methods such as [Srinivasan and Varghese 1999]).

Improved construction of fixed and variable stride tries. Sahni and Kim in a series of papers [Sahni and Kim 2001; 2002; 2003] propose new formulations of the dynamic programming underlying the construction of the fixed stride and variable stride tries in [Srinivasan and Varghese 1999]. The emphasis is on improving the preprocessing time. Both in the fixed stride and in the variable stride cases the user defines an input parameter $k$ that is the depth of the tree. For a a given value of $k$ the searching algorithm and the searching performance is identical to that in [Srinivasan and Varghese 1999]. Thus the other quality measure that need to be measured is the memory consumption. For $k=3$ and variable stride trie, for a table of $35 \mathrm{~K}$ prefixes, the reported storage is 677 Kbytes [Sahni and Kim 2003, Table 4, page 676], which is reasonably close to the storage bound reported in [Srinivasan and Varghese 1999]. With the same data set, a value $k=2$ would lower the search time but push up the storage to 1.8 Kbytes. Thus we can conclude that the storage and search time trade offs are analogous to those in [Srinivasan and Varghese 1999].

Data structures stressing dynamic operations. Sahni and Kim in [Sahni and Kim 2004] give a data structure that improves the update time with respect to the variable stride tries in [Srinivasan and Varghese 1999; Sahni and Kim 2003] but has slower search time and larger storage requirements. In our setting we compare data structures by giving higher priority to search time (within reasonable storage), next in importance is storage and only the last criterion in update time. Thus the AST and the result in [Sahni and Kim 2004] are not comparable because they imply a different ranking of relevant performance measures. Similarly, Lu and Sahni [Lu and Sahni 2005] propose a variant of the B-tree to handle efficiently updates. Experimental data reported cannot be compared directly with ours since the raw timing reported in [Lu and Sahni 2005] comprises accesses in RAM memory, while in our analysis we fit all data in L2-level cache, however we can make use of a cachemiss analysis in [Lu and Sahni 2005, page 17] that estimates a worst case number of cache misses for look up search at $0.5 \log _{2} n$. This number would correspond to memory accesses if the data structure were completely in L2 cache. For $n=32 \mathrm{~K}$ prefixes this would imply a number of about 7 memory accesses. This is far from the AST count of 3 for similar input sizes. This discrepancy is obviously due to the emphasis in improving dynamic operations at expenses of lookup efficiency. Lu and Shani in [Lu and Sahni 2004b] explore the use of red-black trees and prioritysearch trees for solving the longest prefix mach problem (as well as other related problems). Since these are trees with branching factor 2 , they put stress on low

Journal of the ACM, Vol. V, No. N, Month 20YY. 
memory consumption and fast updates at the cost of having to visit paths of length $O(\log n)$ in order to solve IP-lookup queries. Sun, Sahni and Zhao [Sun et al. 2004] propose a general technique for exploiting independent sets in the input data on a multi-processor parallel architecture to speed up update operations.

A very recent data structure Sundström and Larzon [Sundström and Larzon 2005] give a data structure whose main feature is to be able to store large data sets requiring just 4 memory accesses to handle a lookup query. More precisely a data set of 131227 prefixes is stored in $950 \mathrm{~K}$ bytes and a search requires 4 memory access. The update operations on this data set have a worst case cost of 752 memory accesses. The data sets 8,9,10 and 11 in our Table (I) get close to that used in [Sundström and Larzon 2005] in terms of sheer size. We see that we can achieve for those data seta only 3 memory accesses. The Slim-AST achieves also similar storage bounds but cannot handle updates directly. The Fat-AST would supports dynamic operations but uses roughly $50 \%$ more memory on such input data.

Partitioning schemes. Lu, Kiam and Sahni [Lu et al. 2005] propose several schemes for an initial partition of the data set into disjoint subsets. The rationale is to be able to apply for each subset a more traditional data structure (e.g. variable stride tries) whose parameters are optimized on the local input subset. This operation leads in experiments to improve in storage and average search time. Note that this technique is "generic" and as such could be applied directly also to AST's.

Data Structures stressing storage requirements. Elaborating on ideas in [Degermark et al. 1997] Earthon at al. [Eatherton et al. 2004] propose the Tree bitmap data structure that can be adapted to several memory models. For the memory model adopted in this paper (L2 and L1 cache levels) they are able to re-map the tables in [Degermark et al. 1997] so that each tree node access requires only 1 memory access (while in [Degermark et al. 1997]) 4 memory access per node are needed. However the requirement of small memory occupancy results in a tree with higher depth. In experiments a data set of 40,902 prefixes is stored in 312 Kbytes but requires 7 memory accesses in worst case. Moreover the technique in [Eatherton et al. 2004] allows for fast average update time.

Different matching rules. Sahni and $\mathrm{Lu}$ in [Lu and Sahni 2004a] explore a slightly different problem matching rule. Here it is supposed each prefix has an associated priority unrelated to the length and the problem is to return the highest priority prefix matching the destination address of a packet. The emphasis is on good worst case asymptotic bounds on storage and update time. The number of memory accesses is equivalent to the height of the tree which is $O(\log n)$. For $n=32 \mathrm{~K}$ this would imply about 15 memory accesses.

Hashing Based schemes The basic scheme of [Waldvogel et al. 1997] (binary search on levels) is to expand certain prefixes so to obtain a small number of surviving prefix lengths and store all prefixes of the same length and markers for longer prefixes in a hash table. The IP-lookup problem is then solved by a binary search by prefix length. Kim and Sahni improve the basic approach in [Waldvogel et al. 1997] by providing a new dynamic programming formulation whose aim is to reduce storage consumption as well as reducing the number of prefix lengths. Broder and Mitzenmacher [Broder and Mitzenmacher 2001b] give a technique for improving 
the performance of a single hash tables. The use of randomized schemes based on hashing can lead to good average case performance however this is paid for in the difficulty of controlling worst case lookup time (which basically corresponds to the maximum bucket occupancy of one of the hash tables used). Moreover dynamic updates becomes problematic since the event of exceeding the maximum allowed bucket occupancy (for speed each bucket can occupy a single cache-line) requires the complete re-computation of the hash functions and of the hash tables. In order to give a feeling of what can be accomplished with this technique we report an experiment in [Broder and Mitzenmacher 2001b] with a table of 38,816 prefixes showing that the scheme in [Waldvogel et al. 1997] is able to solve queries with two memory accesses, using 4 megabytes, under the assumption that six prefixes can be stored in a single cache line. The scheme in [Broder and Mitzenmacher 2001b] reduces the maximum bucket occupancy to four, uses half of the storage, but requires to visit two buckets in each hash table, thus, requiring in total four memory accesses. In contrast the Fat-AST requires 3 memory accesses, thus is better than [Broder and Mitzenmacher 2001b], for a much smaller memory occupancy.

Hw based methods. There is a quite large portion of the recent research on the IP lookup problem that uses specialized hw architectures different form the hw model adopted in this paper (e.g. [Hasan and Vijaykumar 2005], [Dharmapurikar et al. 2003], [Taylor et al. 2003], [Liu and Lea 2001], [Wuu and Pin 2001]). For this reason we do not comment further on these results.

\section{ON THE STATIC AND DYNAMIC PREDECESSOR PROBLEM}

On the predecessor problem. Consider the following rather general formulation. Let $U=\left[0,2^{w}-1\right]$ be the set of positive integers expressible in binary notation with words of $w$ bits $^{10}$ and let $S \subset U$ be a finite subset of cardinality $n$. The complement set $U \backslash S$ is composed of a collection $I_{S}$ of disjoint open intervals. The universe $U$ is thus partitioned into a collection of intervals $I_{S}$ and points $S$. Let $f$ be a function defined on $U$, with range values in a set $H$, such that $f$ is invariant within each interval of $I_{S}$. The problem is to build at pre-processing time a data structure $D(U, S)$ so to retrieve efficiently $f(q)$ for a query $q \in U$ given on-line. For the static setting the exact nature of the range set $H$ and of the function $f$ is immaterial and we concentrate on the task of searching, given a query $q \in U$, the node in the data structure $D(U, S)$ holding the associated value $f(q)$. Since we can associate the value for points in each interval $I_{S}$ to the left end point of the interval, this query problem is equivalent to the predecessor problem: given $U$ and $S$ as above, for any $x \in U$ find $\max \{y \in S \mid y \leq x\}$.

Solutions to the (static) predecessor problem have performance bounds that are very sensitive to two conditions, the first is the relative size of $n$ with respect to $U$, the second is the maximum amount of storage allowed. We may insist in having just linear storage $O(n)$, or storage polynomial in $n$ or we may allow storage superpolynomial in $n$ (i.e. polynomial in $n$ and $U$ ). Note that, if $n$ is not too small with respect to $U$, technically $n>U^{1 / C}$ for some constant $C>1$, then also $U<n^{C}$ and therefore a polynomial in $n$ and $U$ is automatically a polynomial also in $n$ only.

\footnotetext{
${ }^{10}$ In typical current processors $w=32$.

Journal of the ACM, Vol. V, No. N, Month 20YY.
} 
By using a k-ary tree with $k=U^{\epsilon}$, the resulting tree uses storage $O\left(n U^{\epsilon}\right)$ and the query time is constant. The height of the tree is $\frac{\log U}{\log U^{\epsilon}}=\frac{1}{\epsilon}=O(1)$, while the storage is bounded by $O(n k)=O\left(n U^{\epsilon}\right)$, see e.g. [Eppstein and Muthukrishnan 2001]. Naturally, with the observation above, when $n>U^{1 / C}$ the storage is $O\left(n^{1+\epsilon}\right)$ and the query remains $O(1)$.

The results we will survey try to reduce the storage requirement without increasing too much the query time. The methods we survey are in general dynamic, or can made so with some extra machinery, however, since we are interested in the query time vs. storage performance, we will not dwell on such dynamic features.

Willard [Willard 1983] [Willard 1984] gives data structures (fast y-tries and fast qtries) using storage $O(n)$ achieving query time $O(\min \{\log \log U, \sqrt{\log U}\})$. Stratified trees of van Emde Boas achieve query time $O(\log \log U)$ using storage $O(U)$ in the original paper [van Emde Boas et al. 1977]. Storage was reduced afterwards to $O\left(n U^{\epsilon}\right)$ in [Johnson 1982], and to $O(n)$ in [van Emde Boas 1977] when $n>$ $|U| / \log ^{c}|U|$. Willard in [Willard 2000] lowers the lower limit for ataining linear storage to $\left.n>|U| /(\log U)^{c \cdot \log \log |U|}\right)$. Beame and Fich [Beame and Fich 1999] have shown that the predecessor problem among $n$ input data from an integer universe $U$ can be found using $O(n)$ storage and query time

$$
O\left(\min \left\{\frac{(\log \log U)(\log \log n)}{\log \log \log U}, \sqrt{\frac{\log n}{\log \log n}}\right\}\right),
$$

and even more interestingly they show that with storage limited to polynomial this is essentially the best that can be done for a certain range of relative sizes of $n$ and $U$. They obtain the following static lower bounds: for every $n$ such that $(\log U)^{\omega(1)}<n<U^{o(1)}$, using a number polynomial in $n$ of memory cells of roughly $2^{(\log U)^{c}}$ bits each for $c<1$, a query must use in the worst case $\Omega\left(\frac{\log \log U}{\log \log \log U}\right)$ operations, while with cells of $O(\log U)^{C}$ bits each, $\Omega(\sqrt{\log n / \log \log n})$ operations are needed.

There are several results not as strong as those of Beame and Fich but worth recalling. Raman [Raman 1995] gives a data structure using storage $O(n)$ and performing queries in time $O(\min \{\log \log U, 1+\log n / \log \log U\})$. A randomized method of Anderson [Andersson 1995] uses storage $O(n)$ and has expected query time $O(\sqrt{\log n})$. Subsequently in [Andersson 1996], still using linear storage, the method has been made deterministic and the query time improved to

$$
O\left(\min \left\{\sqrt{\log n}, \quad \log \log U \log \log n, \quad \log \log n+\frac{\log n}{\log \log U}\right\}\right) .
$$

The problem of the optimality of the known bounds on the query time for the predecessor search problem is studied in [Patrascu and Thorup 2006].

Knuth in [Knuth 1973] surveys several bucketing schemes (Tries, Patricia Trees, Digital Search tree) which are all variants of the basic $k$-ary tree approach. All these schemes produce search trees of height $\log _{k} n$, while the expected size of the tree is $O(n k / \ln k)$. A variant discussed in [Knuth 1973] uses an hybrid approach, when a bucket holds less than $s$ data items a standard binary search tree is used. The expected height is reduced to $(1+1 / s) \log _{k} n+\log s$, while the storage is $O(n k / s \ln k+n)$. 
Previous results with distribution dependent guarantees. N-trees defined by Ehrlich [Ehrlich 1981] capture in a formal way the probably older idea of recursive bucketing where the number of buckets depends on the number of data items in each bucket. Results in [Ehrlich 1981] show that the expected construction time is $O(n)$ when the data are drawn uniformly at random from the universe. Later Tamminen [Tamminen 1983] showed that for any data set drawn from a distribution with a bounded and Lebesgue integrable probability density function, the height of the N-tree is bounded by a small constant. This result is interesting since it introduces distribution dependent guarantees beyond the standard uniform distribution. Willard [Willard 1985] gives an $O(\log \log n)$ expected time algorithm to search on data drawn from a smooth distribution.

Comparison of AST and predecessor search data structures. The AST doe not have provable worst case bounds that are competitive with the above mentioned structures for predecessor search. However experiments in [Pellegrini and Vecchiocattivi 2001] have shown that the AST trees have storage and depth tradeoffs (measured in number of nodes of the tree and in the longest path) that are significantly superior to k-trees, n-trees and van Emde Boas trees.

\section{ACKNOWLEDGEMENTS}

We thank Giogio Vecchiocattivi who contributed to testing an early version of the AST data structure [Pellegrini et al. 2002], and the participants of the miniworkshop on routing (held in Pisa in March 2003) for many interesting discussions.

\section{CONCLUSIONS AND OPEN PROBLEMS}

Preliminary experiments with AST give encouraging results when compared with published results for state of the art methods, however fine tuning of the AST requires choosing properly some user defined parameters and we do not have at the moment general rules for this choice. How to find a theoretical underpinning to explain the AST good empirical performance is still an open issue for further research.

\section{REFERENCES}

Andersson, A. 1995. Sublogarithmic searching without multiplications. In 36th Annual Symposium on Foundations of Computer Science: October 23-25, 1995, Milwaukee, Wisconsin, IEEE, Ed. IEEE Computer Society Press, 1109 Spring Street, Suite 300, Silver Spring, MD 20910, USA, 655-663.

Andersson, A. 1996. Faster deterministic sorting and searching in linear space. In 37th Annual Symposium on Foundations of Computer Science. IEEE, Burlington, Vermont, 135-141.

BEAmE AND Fich. 1999. Optimal bounds for the predecessor problem. In STOC: ACM Symposium on Theory of Computing (STOC).

Blum, A., Jiang, T., Li, M., Tromp, J., And Yannakakis, M. 1994. Linear approximation of shortest superstrings. Journal of the ACM 41, 4 (July), 630-647.

Broder, A. Z. and Mitzenmacher, M. 2001a. Using multiple hash functions to improve IP lookups. In INFOCOM. 1454-1463.

Broder, A. Z. and Mitzenmacher, M. 2001b. Using multiple hash functions to improve IP lookups. In IEEE Computer and Communications Societies (INFOCOM). Vol. 3. 1454-1463.

Buchsbaum, A. L., Fowler, G. S., Krishnamurthy, B., Vo, K.-P., and Wang, J. 2003. Fast prefix matching of bounded strings. In Proceedings of Alenex 2003.

Journal of the ACM, Vol. V, No. N, Month 20YY. 
Cheung, G. And McCanne, S. 1999. Optimal routing table design for IP address lookups under memory constraints. In INFOCOM (3). 1437-1444.

Crescenzi, P., Dardini, L., And Grossi, R. 1999. IP address lookup made fast and simple. In European Symposium on Algorithms. 65-76.

Degermark, M., Brodnik, A., Carlsson, S., And Pink, S. 1997. Small forwarding tables for fast routing lookups. In SIGCOMM. 3-14.

Dharmapurikar, S., Krishnamurthy, P., and Taylor, D. E. 2003. Longest prefix matching using bloom filters. In Conference on Applications, Technologies, Architectures, and Protocols for Computer Communication (SIGCOMM). ACM Press, 201-212.

Eatherton, W., Varghese, G., And Dittia, Z. 2004. Tree bitmap: Hardware/software IP lookups with incremental updates. Computer Communication Review 34, 2, 97-122.

Ehrlich, G. 1981. Searching and sorting real numbers. Journal of Algorithms 2, 1 (Mar.), 1-12.

Eppstein, D. And Muthukrishnan, S. 2001. Internet packet fileter management and rectangle geometry. In Proceedings of the 12th Annual Symposium on Discrete Algorithms. ACM Press, New York, NY, USA, 827-835.

Ergun, Sahinalp, Sharp, and Sinha. 2001. Biased skip lists for highly skewed access patterns. In ALENEX: International Workshop on Algorithm Engineering and Experimentation, LNCS 2153. 216-229.

Feldmann, A. And Muthukrishnan, S. 2000. Tradeoffs for packet classification. In Proceedings of INFOCOM, volume 3. IEEE, 1193-1202.

Geraci, F., Pellegrini, M., Pisati, P., and Rizzo, L. 2005. Packet classification via improved space decomposition techniques. In Proceedings of INFOCOM 2005. 24th Annual Joint Conference of the IEEE Computer and Communications Societies (March 13-17). Vol. 1. 304312.

Gupta, P., Prabhakar, B., And Boyd, S. P. 2000. Near optimal routing lookups with bounded worst case performance. In INFOCOM (3). 1184-1192.

Hasan, J. And ViJaYkumar, T. N. 2005. Dynamic pipelining: Making IP-lookup truly scalable. ACM SIGCOMM Computer Communication Review 35, 4, 205-216.

Ionnnidis, I., Grama, A., And Atallah, M. J. 2005. Adaptive data structures for IP lookups. ACM Journal of Experimental Algorithms 10.

Johnson, D. B. 1982. A priority queue in which initialization and queue operations take $O(\log (\log (D))$ time. Mathematical Systems Theory 15, 295-309.

Kaplan, H., Molad, E., And TaRJan, R. E. 2003. Dynamic rectangular intersection with priorities. In Proceedings of the thirty-fifth ACM symposium on Theory of computing. ACM Press, 639-648.

Knuth, D. E. 1973. The Art of Computer Programming, Vol.3 - Sorting and Searching. Addison-Wesley (Reading MA).

Lampson, B. W., Srinivasan, V., and Varghese, G. 1999. IP lookups using multiway and multicolumn search. IEEE/ACM Transactions on Networking 7, 3, 324-334.

LiU, Y.-C. And LEA, C.-T. 2001. Fast IP table lookup and memory reduction. In IEEE Workshop on High Performance Switching and Routing. Dallas, TX, USA, 228-232.

LU, H., KIm, K. S., AND SAHNi, S. 2005. Prefix- and interval-partitioned dynamic IP router-tables. IEEE Transactions on Computers 54, 5, 545-557.

Lu, H. And SAhni, S. 2004a. Dynamic IP router-tables using highest-priority matching. In IEEE Symposium on Computers and Communications. 858-863.

Lu, H. AND SAHNI, S. 2004b. $O(\log n)$ dynamic router-tables for prefixes and ranges. IEEE Transactions on Computers 53, 10, 1217-1230.

Lu, H. And SAhni, S. 2005. A b-tree dynamic router-table design. IEEE Transactions on Computers 54, 7, 813-824.

McKeown, N. 1999. Hot interconnects tutorial slides. Stanford University, available at http://klamath.stanford.edu/talks/.

MORRISON, D. R. 1968. PATRICIA - practical algorithm to retrieve information coded in alphanumeric. Journal of ACM 15, 4 (Oct.), 514-534.

Journal of the ACM, Vol. V, No. N, Month 20YY. 
Mulmuley, K. 1993. Computational Geometry, an Introduction through Randomized Algorithms. Prentice Hall.

Nilsson, S. And Karlsson, G. 1999. IP-Address Lookup Using LC-Tries. IEEE Journal on Selected Areas in Communications.

Patrascu, M. And Thorup, M. 2006. Time-space trade-offs for predecessor search. In STOC '06: Proceedings of the thirty-eighth annual ACM symposium on Theory of computing. ACM Press, New York, NY, USA, 232-240.

Pellegrini, M., Fusco, G., And Vecchiocattivi, G. 2002. Adaptive stratified search trees for ip table lookup. Tech. Rep. TR IIT 22/2002, Istituto di Informatica e Telematica del CNR (IIT-CNR), Pisa,Italy. November.

Pellegrini, M. and Vecchiocattivi, G. 2001. Empirical study of search trees for ip addressing look up. Tech. Rep. TR IMC B4-01-13, Istituto di Matematica Computazionale del CNR, Area della Ricerca, Pisa, Italy. November. Available at www.iit.cnr.it/staff/marco.pellegrini.

Pugh, W. 1990. Skip lists: A probabilistic alternative to balanced trees. Communications of the ACM 33, 6 (June), 668-676.

RAMAN, R. 1995. Improved data structures for predecessor queries in integer sets.

SAHni, S. AND KIM, K. S. 2001. Efficient construction of fixed-stride multibit tries for IP lookup. In 8th IEEE Workshop on Future Trends of Distributed Computer Systems (FTDCS). IEEE Computer Society, Bologna, Italy, 178-184.

SAHNI, S. AND KIM, K. S. 2002. Efficient construction of variable-stride multibit tries for IP lookup. In Symposium on Applications and the Internet (SAINT). IEEE Computer Society, Nara City, Japan, 220-229.

SAHNI, S. AND KIM, K. S. 2003. Efficient construction of multibit tries for IP lookup. IEEE/ACM Transactions on Networking 11, 4, 650-662.

Sahni, S. And Kim, K. S. 2004. Efficient dynamic lookup for bursty access patterns. International Journal on Foundations of Computer Science 15, 4, 567-591.

Sharp, J., Ergun, F., Mittra, S., Sahinalp, C., And Sinha, R. 2001. A dynamic lookup scheme for bursty access patterns. In Proceedings of the Twentieth Annual Joint Conference of the IEEE Computer and Communications Societies (INFOCOM-01). IEEE Computer Society, Los Alamitos, CA, 1444-1453.

SkLOwER, K. 1991. A tree-based packet routing table for berkeley unix. In USENIX Winter 1991. 93-104.

SRinivasan, V. AND VARghese, G. 1999. Fast address lookups using controlled prefix expansion. ACM Transactions on Computer Systems, 1-40.

Sun, X., Sahni, S. K., And ZhaO, Y. Q. 2004. Fast update algorithm for IP forwarding table using independent sets. In High Speed Networks and Multimedia Communications (HSNMC 2004), Z. Mammeri and P. Lorenz, Eds. Lecture Notes in Computer Science, vol. 3079. Springer, Toulouse, France, 324-335.

SUNDSTRÖM, M. AND LARZON, L.- $\AA$. 2005. High-performance longest prefix matching supporting high-speed incremental updates and guaranteed compression. In IEEE Computer and Communications Societies (INFOCOM). Vol. 3. 1641-1652.

Tamminen, M. 1983. Analysis of $N$-trees. Information Processing Letters 16, 3 (Apr.), 131-137.

Taylor, D. E., Lockwood, J. W., Sproull, T. S., Turner, J. S., and Parlour, D. 2003. Scalable IP lookup for internet routers. IEEE Journal on Selected Areas in Communications 21, 4.

Thorup, M. 2003. Space efficient dynamic stabbing with fast queries. In Proceedings of the 35th Annual ACM Symposium on Theory of Computing, June 9-11, 2003, San Diego, CA, USA. ACM, 649-658.

VAN Emde Boas, P. 1977. Preserving order in a forest in less than logarithmic time. Information Processing Letters 6, 80-82.

van Emde Boas, P., KaAs, R., And Zijlstra, E. 1977. Design and implementation of an efficient priority queue. Mathematical Systems Theory 10, 99-127.

Waldvogel, M., Varghese, G., Turner, J., and Plattner, B. 1997. Scalable high speed IP routing lookups. In SIGCOMM. 25-36.

Journal of the ACM, Vol. V, No. N, Month 20YY. 
Warkhede, P. R., Suri, S., And Varghese, G. 2004. Multiway range trees: Scalable IP lookup with fast updates. Computer Networks 44, 3, 289-303.

Willard, D. E. 1983. Log-logarithmic worst-case range queries are possible in space $\Theta(N)$. Information Processing Letters 17, 2 (24 Aug.), 81-84.

Willard, D. E. 1984. New trie data structures which support very fast search operations. Journal of Computer and System Sciences 28, 3 (June), 379-394.

Willard, D. E. 1985. Searching unindexed and nonuniformly generated files in $\log \log N$ time. SIAM Journal on Computing 14, 4 (Nov.), 1013-1029.

Willard, D. E. 2000. Examining computational geometry, Van Emde Boas trees, and hashing from the perspective of the fusion tree. SIAM Journal on Computing 29, 3 (June), 1030-1049.

WuU, L.-C. AND PIN, S.-Y. 2001. A fast IP lookup scheme for longest-matching prefix. In International Conference on Computer Networks and Mobile Computing (ICCNMC). IEEE Computer Society, 407-412.

Received Month Year; revised Month Year; accepted Month Year 